\title{
Monitoring of Indoor Airflows with a New Two-Dimensional Airflow Sensor
}

\author{
Yuanchen Wang, Christian Lodroner, Michael Müller, and Konstantinos Stergiaropoulos \\ University of Stuttgart, Institute for Building Energetics, Thermotechnology and Energy Storage, 70569 Stuttgart, Germany
}

\begin{abstract}
Although airflow is invisible, it has a big influence on the indoor environment. An incorrectly planned HVAC systems can lead to draught and thermal discomfort in occupied zones. Since the commissioning tests required after the installation of HVAC systems are generally performed without occupancy, the tests results do not always accurately represent the airflow that occurs during ordinary usage. The airflow needs to be continuously monitored and controlled by an intelligent HVAC system. The aim of this study is to develop a new two-dimensional airflow sensor for the monitoring of indoor airflow, which can also indicate the flow direction. Several of these sensors can be placed in a planar sensor array, by which a two-dimensional section of the flow field is created. By recording data from several of these arrays simultaneously, an image of the three-dimensional flow could be acquired. The prototype of the sensor, which is made by Hahn-Schickard Society for Applied Research is currently being validated at the Institute for Building Energetics, Thermotechnology and Energy Storage. When the development is completed, it will greatly contribute to the control strategies of HVAC systems.
\end{abstract}

\section{Introduction}

\subsection{Background and motivation}

People spend a large amount of their lifetime in indoors. As a result of the stricter requirements of the European Energy Performance of Buildings Directive (EPBD) [1] on the airtightness of the building, the free exchange of air due to infiltration is very low. This leads to an increasing use of HVAC systems to ensure the compliance with indoor air quality and thermal requirements. Due to the indoor comfort and energy performance requirements, an intelligent control of the buildings is necessary [2].

In modern buildings, various parameters such as temperature, humidity, and $\mathrm{CO}_{2}$-concentration are measured with sensors and used to control HVAC systems. The measuring of the airflows is not monitored in most cases, even though according to the international standard ISO 7730 [3] draught is the most common cause of local discomfort in buildings. Fanger et al. [4] have suggested Equation 1 for calculating the draught rating (DR) to describe the percentage of people dissatisfied due to draught:

$$
D R=\left(34-t_{a}\right)(\bar{v}-0.05)^{0.62}\left(0.37 \bar{v} T_{u}+3.14\right)
$$

where: $t_{\mathrm{a}}$ is the local air temperature $\left({ }^{\circ} \mathrm{C}\right) ; \bar{v}$ is the local mean air speed $\left(\mathrm{m} \mathrm{s}^{-1}\right)$; and $T_{u}$ is the local air turbulence (\%) defined as the ratio of the standard deviation of the local air speed to the local mean air speed.

Equation 1 shows that the risk of draught is not only dependent on the room temperature, but also on the local air speed and the turbulence intensity. These are usually predicted during the planning stage by simulation, or measured during commissioning, maintenance or servicing.

However, the airflows are mostly transient and dependent on the occupation of the room. Therefore, a limited amount of measurements outside of conditions during occupation of the room is not sufficient. When the HVAC systems are operated under transient conditions $[5,6]$, the prediction is even more difficult.

Furthermore, the evaluation by ISO 7730 was developed for relatively homogeneous turbulence patterns. Rare peaks or very unsteady airflows will be smoothed out due to averaging and are not taken into account for the evaluation. Disturbances of the comfort due to unsteady airflows are thereby systematically underestimated by ISO 7730 [7].

For these reasons, a continuous measurement over time should be considered to comprehensively evaluate airflows in indoor environments. ISO 7730 recommends the use of omnidirectional anemometers for thermal comfort measurements. However, for continuous measurements in real time, information about the direction of the airflow can be very helpful for the analysis of indoor environments, as well as HVAC diagnostics and controls.

\subsection{State of the art}

ISO 7726 [8] lists three different measurement principles for airflow measurements that are able to

\footnotetext{
* Corresponding author: yuanchen.wang@igte.uni-stuttgart.de
} 
acquire information about the direction of the airflow as well:

- Optical anemometers: The Laser Doppler Anemometer (LDA) is often used for high-precision reference measurement for sensor calibration. Particle Image Velocimetry (PIV) is often used in research to determine the velocity field in a flow. They can also be used to measure airflow in buildings [9, 10]. However, these methods require seeding particles interspersed in the air. Therefore, they are not suited for continuous measuring during the occupation of a room. Additionally, their high price and complex operation limit their use for measurements of indoor airflows.

- Ultrasonic anemometers are often used in meteorology for measurements of horizontal wind speed and direction. For accurately measuring indoor airflows in the range of $0.05 \mathrm{~m} \mathrm{~s}^{-1}$ to $1 \mathrm{~m} \mathrm{~s}^{-1}$ a special three-dimensional ultrasonic anemometer was used by Koskela et al. [11, 12]. Such ultrasonic anemometers are very expensive, which makes their use in a sensor array especially costly.

- Thermal anemometers e.g. the hot wire or hot sphere anemometers have a high measurement accuracy, even at low air speeds. They are the most common type of sensor used for indoor airflow measurements. The hot sphere anemometer is omnidirectional and can only measure the speed of the airflow. Certain designs of hot wire anemometer (e. g. Dual-sensor Probes or Triple-sensor Probes) are able to measure the airflow in two or three dimensions. However, the price of such anemometers strongly increases with the ability to measure in multiple dimensions. Additionally, measurements in multiple dimensions are limited to certain sectors [13], as each wire acquires only the magnitude of its corresponding velocity component. Another disadvantage of thermal anemometers is that they are fragile, even with protection baskets, which makes a use in rooms during occupation difficult.

As shown, there is demand for an airflow sensor that is able to measure the air speed, as well as direction, which is low-priced, miniature, light, mobile and robust. Therefore, a new sensor which is based on the Seebeckeffect (thermoelectric effect) was developed by the project partners Institute for Building Energetics, Thermotechnology and Energy Storage at the University of Stuttgart (IGTE) and Hahn-Schickard Society for Applied Research in Villingen-Schwenningen (HS-VS). As the three-dimensional measurement of airflows is complicated, the project aims to develop a twodimensional sensor first. The technology and experience from this project could be used to implement threedimensional airflow measurements for future studies.

\section{Sensor description}

The core part of the developed sensor is a silicon-nitridemembrane with an edge length of $600 \mu \mathrm{m}$, which spans a silicon chip. Due to its dimensions and material properties, the membrane has a low heat capacity and thermal conductivity and thereby, a low thermal inertia.

A heating element made of polysilicon and surrounded by four thermopiles is placed on the membrane. The thermopiles are arrays of serially connected thermoelements of polysilicon and aluminum that are contacted to the membrane and the silicon chip (Figure1). When there is a temperature difference between the contact points on the bulk silicon material and the contact points on the thermally insulated membrane, the SeebeckEffect leads to a thermoelectric voltage. The thermopiles used in the sensor provide a voltage of 2,2 mV per Kelvin temperature difference.

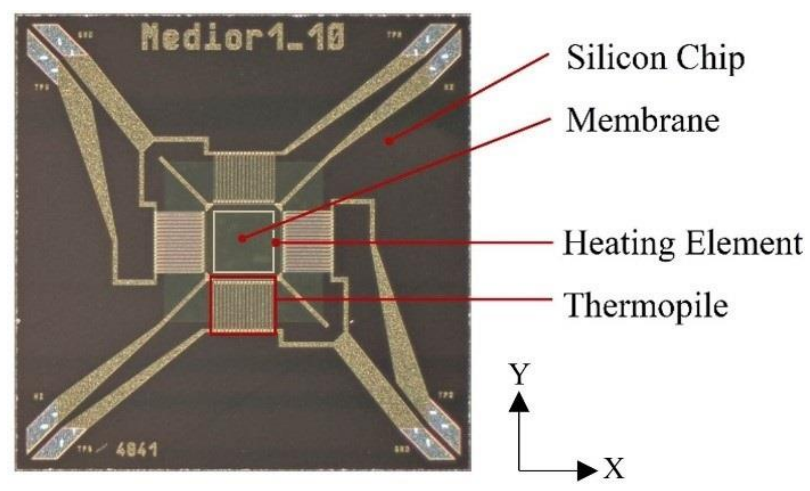

Fig. 1: Manufactured sensor chip [14]

During operation, the heating element is supplied with an electric power of 5 to $10 \mathrm{~mW}$ which leads to an overtemperature of 50 to $80 \mathrm{~K}$. This causes a temperature field around the heating element. In stationary air, this temperature field is symmetrical (solid curve in Figure 2) and the thermoelectric voltages of the upstream and downstream thermopiles are equal. When the air flows over the sensor chip, the temperature field is shifted along the direction of the airflow (dotted curve in Figure 2) which results in different voltages across the two thermopiles. The voltage difference between the two thermopiles is no longer zero, but varies depending on the air speed in this direction. The perpendicular installation of four thermopiles around the heating element enables the measuring of the airflow in $\mathrm{X}$ - and $\mathrm{Y}$-direction.

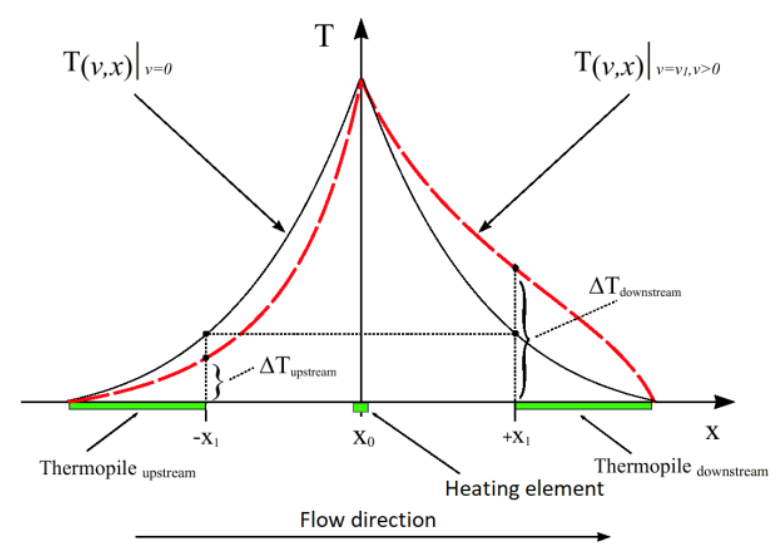

Fig. 2. Schematic temperature profile in the sensor plane [14]

The sensor chip is mounted into a plastic casing, which has the purpose of restricting the airflow along the $\mathrm{XY}$ - plane and directing the airflow over the sensor chip. The sensor probe has a plug connection with the 
transducer. This transducer is based on a micro-processor (PSoC® 5), that is capable of regulating the heating element and to measure and digitise the voltage output of the thermopiles. The correlation between the digital output signals and the air speed is determined by the following calibration procedure.

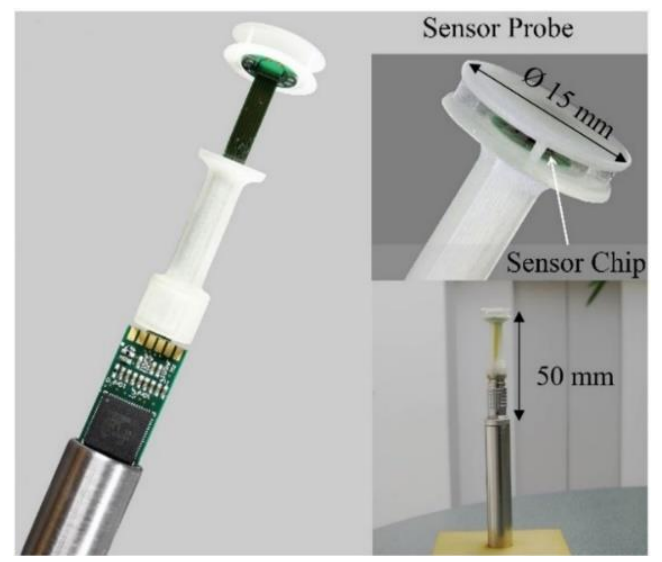

Fig. 3: Picture of the sensor probe [14]

\section{Sensor calibration}

\subsection{Calibration procedure}

Since the sensor is designed to measure low air velocities within the range $0.05-1 \mathrm{~m} \mathrm{~s}^{-1}$, a precise test bench for calibration in this range is necessary. A wind tunnel present at the IGTE can produce airflows from $0.5 \mathrm{~m} \mathrm{~s}^{-1}$ to $30 \mathrm{~m} \mathrm{~s}^{-1}$. This alone is not sufficient for a precise calibration at low velocities. For that reason, a special flow channel was designed and built [15]. The flow channel uses compressed air to produce laminar airflows within the range $0.05-0.74 \mathrm{~m} \mathrm{~s}^{-1}$. For the remaining range the wind tunnel is used.

The calibration procedure complies with the specifications mentioned in the standard ISO 16622 [16]. For the calibration, reference measurements are first done with a precise omnidirectional thermal anemometer (ThermoAir6, 0.015-1 $\mathrm{m} \mathrm{s}^{-1}$ ) to determine the correlation between the control variables of the test benches (mass flow rate for the flow channel, rotational frequency for the fan in the wind tunnel) and the produced air speed. Afterwards, the reference anemometer is substituted with the sensor that needs to be calibrated. To set the angle of attack, the sensor is mounted on a motorized rotation stage.

Room temperature and airflow temperature inside the flow channel during calibration are monitored and regulated to $23{ }^{\circ} \mathrm{C} \pm 0.5 \mathrm{~K}$, the static pressure is usually observed within $(964 \pm 4)$ mbar. The turbulence intensity measured with the reference anemometer in the flow channel is $0.85 \%$ for the lowest setting of $0.05 \mathrm{~m} \mathrm{~s}^{-1}$ and $0.22 \%$ for the highest setting of $0.74 \mathrm{~m} \mathrm{~s}^{-1}$. The turbulence intensity in the wind tunnel is approximately $1.45 \%$. For each selected reference speed and angle of attack the measurement is done for two minutes, with a rate of ten measurements per second.
As described in chapter 2, there is a correlation between air speed and the output signals. To determine this correlation, measurements are done with the sensor in its four major directions $X_{-} \Rightarrow X_{+}, X_{+} \Rightarrow X_{-}, Y_{-} \Rightarrow Y_{+}$and $Y_{+} \Rightarrow Y_{-}$at different air speeds (see positioning in Figure 4). The magnitudes of the velocities in the directions $X_{-} \Rightarrow X_{+}$ and $Y_{-} \Rightarrow Y_{+}$are given a positive sign, and the magnitudes of the velocities in the opposite directions $X_{+} \Rightarrow X$. and $Y_{+} \Rightarrow Y_{-}$are given a negative sign.

The output signals of the four thermopiles are referred to as $U_{X+}, U_{X-}, U_{Y+}$, and $U_{Y-\text {. }}$ The output differences of the thermopile pairs are referred to as $\Delta U_{X}$ and $\Delta U_{Y}$ and are calculated as follows:

$$
\begin{aligned}
& \Delta U_{X}=U_{X^{+}}-U_{X-} \\
& \Delta U_{Y}=U_{Y^{+}}-U_{Y^{-}}
\end{aligned}
$$

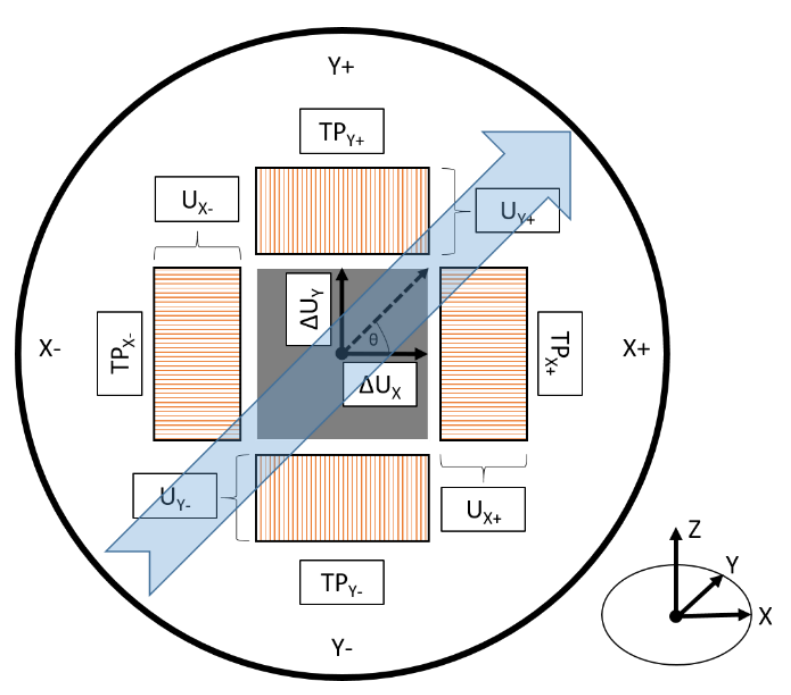

Fig. 4: Layout of the thermopiles and heating element

The output signals $\left(\Delta U_{X}\right.$ and $\left.\Delta U_{Y}\right)$ of the sensor are mapped onto their corresponding velocities. Using these output signals, two calibration curves are created (solid curves in Figure 5).

The two curves follow a similar pattern. As a result of manufacturing tolerances however, the thermopiles cannot be positioned exactly at the same distance around the heating element. The resulting unequal temperature distribution causes an offset between the two curves on the output signal axis.

Another difficulty in the calibration is the decrease of the overtemperature of the heating element with increasing air speed, since the sensor is supplied with constant power. This has an effect on the calibration curves, which is why the velocity components $\left(v_{x}, v_{y}\right)$ cannot be calculated separately from the signal outputs $\left(\Delta U_{X}, \Delta U_{Y}\right)$. For any airflow angle outside the four major directions, this would result in an error, since the magnitude of the velocity in the XY-plane is greater than each of its velocity components. A higher velocity however corresponds to a lower overtemperature and thus also to a different signal value. For this reason, a different evaluation method is used. 
For this method, the curves are shifted as follows, using the values $U_{X}{ }^{0}$ and $U_{Y}{ }^{0}$ of the output signal at zero flow (dotted curve in Figure 5):

$$
\begin{aligned}
& \Delta U_{X}{ }^{*}=\Delta U_{X}-U_{X}{ }^{0} \\
& \Delta U_{Y}{ }^{*}=\Delta U_{Y}-U_{Y}{ }^{0}
\end{aligned}
$$

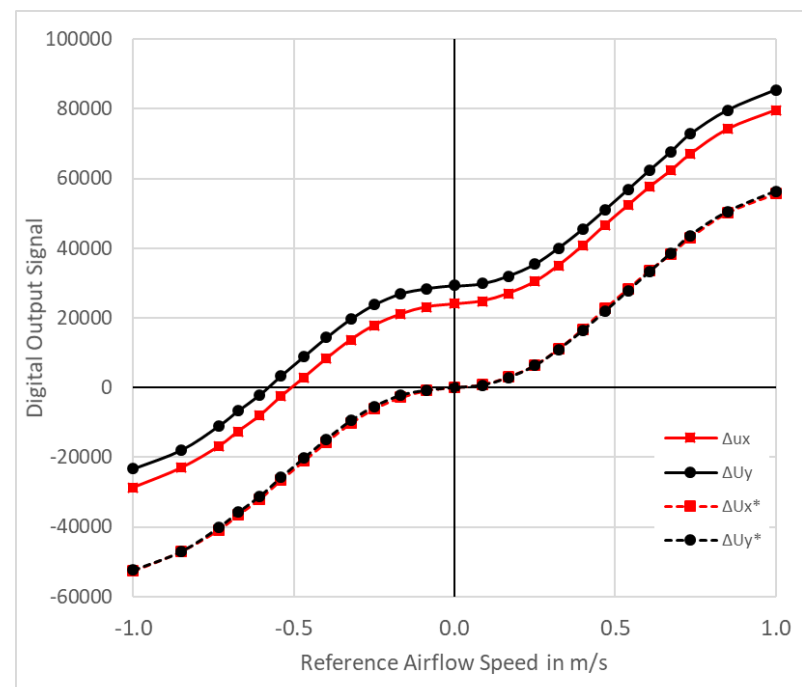

Fig. 5: Shift of the calibration curves; Slight difference after adjustment

The remaining difference between the shifted curves is small enough to take the mean for further evaluation, resulting in an average calibration curve that can be used for all airflow directions. In order to acquire a single value that can be matched with the calibration curve, the output signals are interpreted as a vector and the magnitude of this signal vector is calculated:

$$
U_{\text {sum }}=\sqrt{\left(\Delta U_{X}^{*}\right)^{2}+\left(\Delta U_{Y}^{*}\right)^{2}}
$$

Using this value in the calibration curve yields an airflow speed that corresponds to the correct overtemperature of the heating element.

Since the signal curves of the two thermopile pairs under rotation are sine/cosine shaped, the angle at which the airflow attacks the sensor can be approximated from the shifted output signals using the trigonometric function shown below. The result is the direction of the airflow in degrees.

$$
\begin{gathered}
\theta=180^{\circ}-\operatorname{sgn}\left(\Delta U_{Y}^{*}\right) \frac{180^{\circ}}{\pi} \arccos \left(-\frac{\Delta U_{Y}^{*}}{U_{\text {sum }}}\right) \\
\operatorname{sgn}(x)=\left\{\begin{array}{rl}
1 & \text { if } x \geq 0 \\
-1 & \text { if } x<0
\end{array}, \quad x \in \mathbb{R}\right.
\end{gathered}
$$

As can be seen in Figure 5, the correlation of airflow speed and output signal is not linear. Approximating the correlation accurately with a simple equation has proved difficult. Data fitting yielded good results at intermediate air speeds, but left larger deviations at low and high air speeds. Therefore, in this research a lookup table is used to store the calibration data. Since there are a sufficient amount of data points, linear interpolation is done between the points.

\subsection{Measurement uncertainty}

To calculate the measurement uncertainty of the sensor, many different influences were taken into account. The influences on the measurement uncertainty are shown in the fishbone diagram (Figure 6). Since the reference sensor is substituted with the sensor being calibrated, the reproducibility of the calibration bench plays an important role. The velocity distribution inside the flow channel, as well as the difference in the blockage effect due to different dimensions of the sensors were considered. Some influences were very small and as such neglected, e.g. the influences of humidity, the resolution of the sensor signal and the accuracy of the analog to digital converter.

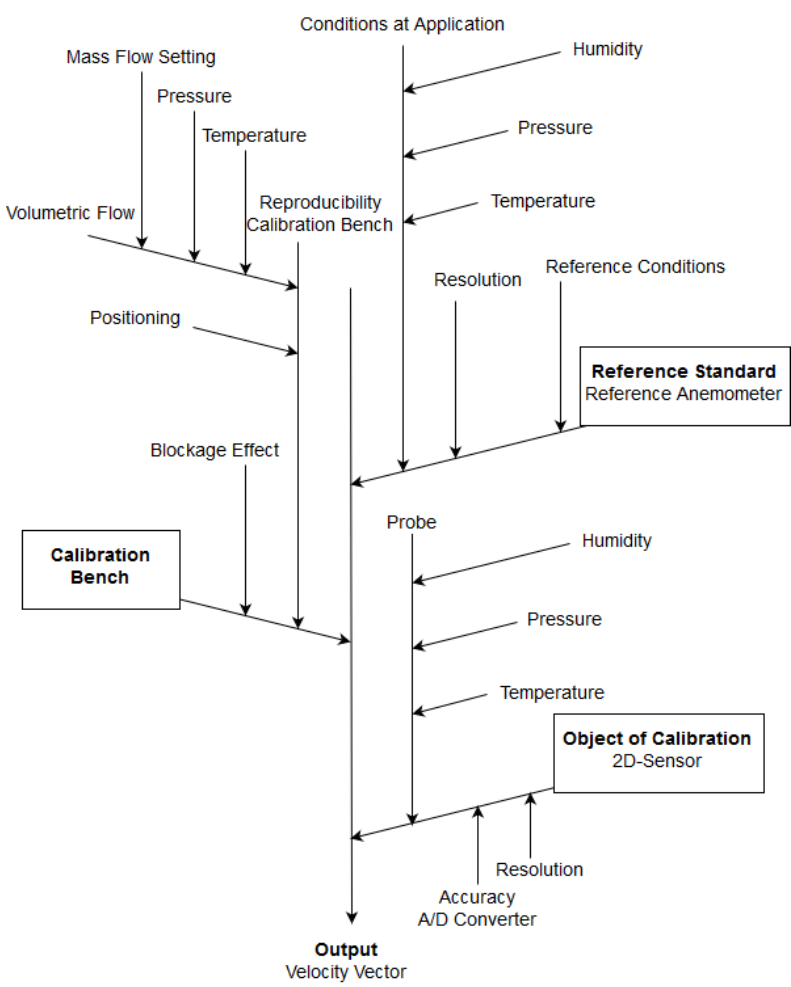

Fig. 6: Fishbone diagram to determine influences on the measurement uncertainty

Unlike an omnidirectional anemometer, the sensor is able to measure the directional information of the airflow as well. The expanded measurement uncertainty for the air speed measurement was determined to be $\pm 4.8 \%$ (of reading) $+1.1 \%$ (of full scale) for a confidence level of $95 \%$. This is valid for all airflows inside the XY-plane of the sensor. The expanded measurement uncertainty for the directional measurement is $\pm 6^{\circ}$ for a confidence level of $95 \%$.

\section{Experiments in a test chamber}

To verify the function of the developed sensor in application-oriented scenarios, two experiments were carried out under isothermal conditions in an environmental test chamber with dimensions of $4.7 \times 4.2 \times 3.6 \mathrm{~m}^{3} \quad$ (length $\times$ width $\times$ height $)$, as shown in Figure 7. In each experiment, the flow pattern was 
visualized using fog and video recording for comparison with the measurement results.

\subsection{Airflow in a ventilated room}

In this experiment, an inlet jet was blown from a jet nozzle, which was installed on a sidewall at a height of $3.3 \mathrm{~m}$. The mean air supply speed was $7.1 \mathrm{~m} \mathrm{~s}^{-1}$. The inlet air temperature during the experiments was equal to the room air temperature within the range $23{ }^{\circ} \mathrm{C} \pm 0.5 \mathrm{~K}$. The outlet opening in the floor was $0.8 \times 0.8 \mathrm{~m}^{2}$.

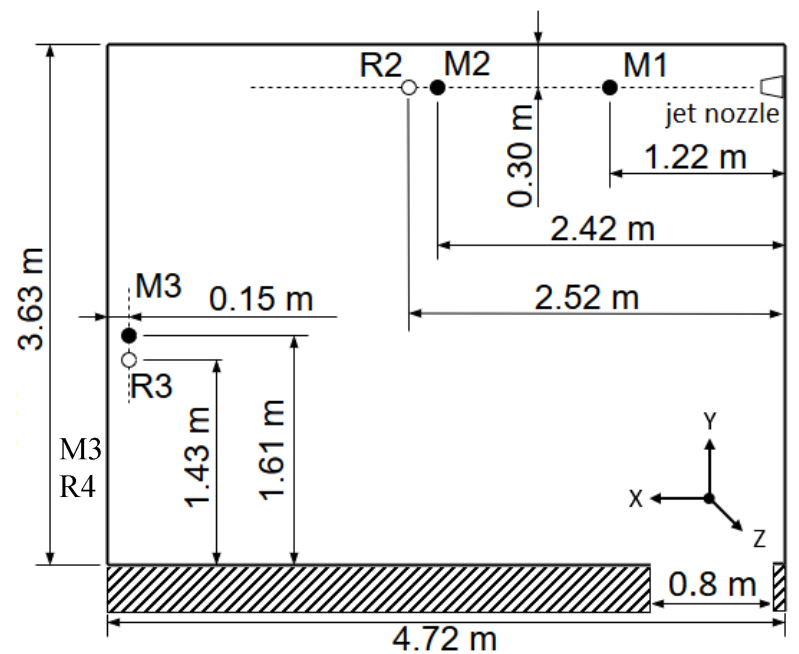

Fig. 7: Placement of the sensors in the test chamber (Side view: the measurement plane is located in the middle section of the room)

For this experiment, three two-dimensional sensors were used. These sensors were set up to measure the speed and direction of the airflow in the XY-plane shown in Figure 7 . However, the z-axis component of the velocity may interfere with measurements. Considering that, the two-dimensional sensors were mounted along the expected airflow path, where the flow direction is well defined and the flow velocity in the z-axis direction should be low enough to be neglected.

The sensor closest to the jet nozzle (M1) is an analog sensor of the first generation of the research project. This sensor can measure velocities up to $3 \mathrm{~m} / \mathrm{s}$. The other two sensors (M2 and M3) are digital sensors with a measuring range of up to $1 \mathrm{~m} / \mathrm{s}$. Reference measurements were done using omnidirectional thermal anemometers (R2 and R3, TSI 8475, 0.05-1 $\mathrm{m} \mathrm{s}^{-1}$ ). A reference measurement at position 1 was not done, as the air speed at this point exceeded the maximum measuring range of the reference sensors of $1 \mathrm{~m} / \mathrm{s}$. The measurements were done over ten minutes, with a rate of ten measurements per second for each sensor.

It should be noted that the air speeds measured with the reference anemometers include the z-axis component of the airflow, which has to be considered in the comparison with the two-dimensional sensors. In order to enable a complete analysis, a CFD-Simulation was carried out. The simulation model used to predict the airflow in the environmental test chamber was a 3D steady simulation in the commercial software ANSYS-Fluent
19.1, using a double precision solver with a RNG k- $\varepsilon$ turbulence model. The number of cells was set to 2 million. Since during the experiment the inlet air almost had the same temperature as the air of the room, the walls in the simulation were set to as adiabatic boundary conditions, in order to simplify the simulation process.

\subsection{Heat plume}

The experiment with the jet zone is an example for an application in ventilated rooms. In order to test the ability of the sensor to measure airflows in heat plumes, another experiment was carried out. For this experiment, an electrical radiator was placed in the middle of the test chamber and the two-dimensional sensor (Figure 9, black dot) was placed above it. An omnidirectional anemometer (Figure 9, white dot) was used again as a reference device and the two sensors were placed in the expected airflow path.

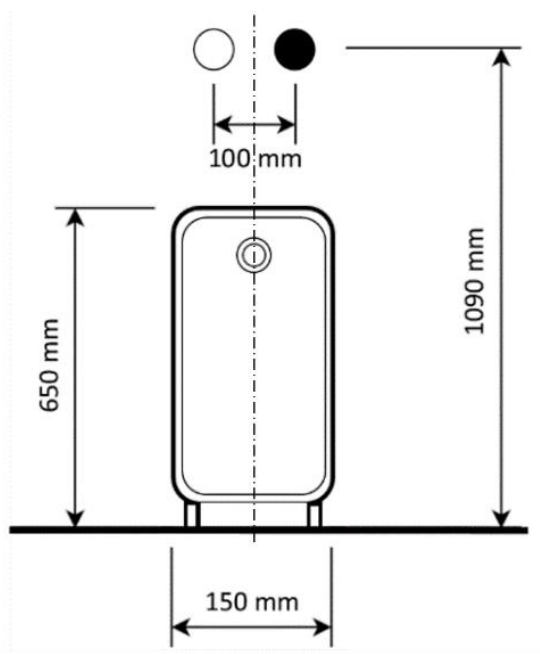

Fig. 9: Drawing of the radiator and the positioning of the sensors

At the start of the experiment, the radiator was at room temperature. It was then supplied with an electric power of $400 \mathrm{~W}$ which resulted in a heat plume developing over time. The airflow was measured by each sensor over ten minutes with a rate of ten measurements per second.

\section{Results and discussion}

\subsection{Airflow in a ventilated room}

\subsubsection{Simulation results}

Figure 10 shows the simulated flow pattern and velocity distribution in the middle section of the room. As expected, the airflow path of the inlet air follows the ceiling and the sidewall. Because of the averaging process of the Reynolds-Averaged Navier-Stokes simulation, there is no turbulence visible in the main flow. The simulation was carried out three-dimensionally. Since the setup of the experiment was symmetrical, the zcomponents of the simulated velocities were negligibly 
low. Therefore, the simulation can be used for a comparison with the measurement results of the twodimensional sensors.

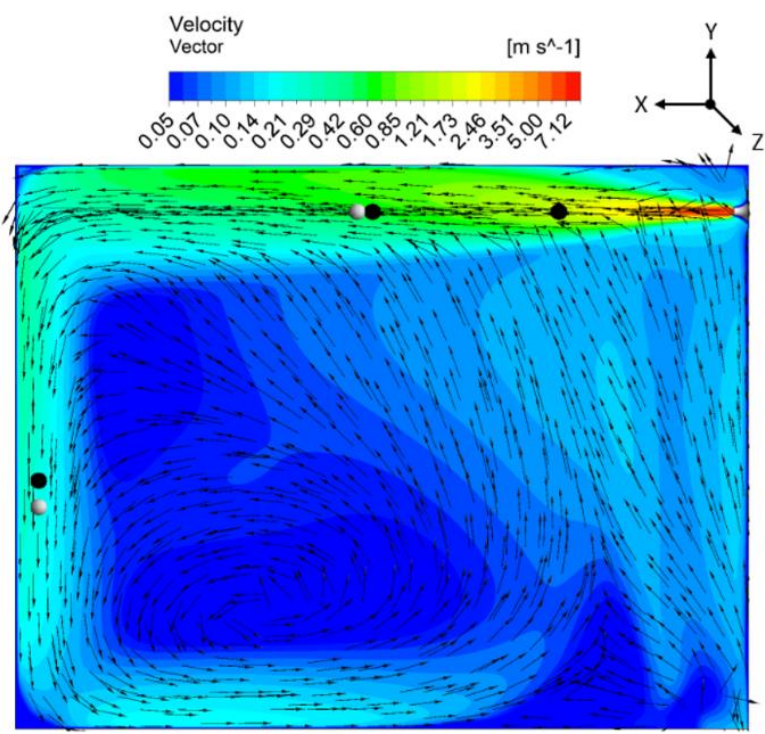

Fig. 10: Simulated flow pattern and velocity distribution

\subsubsection{Airflow visualization with fog}

The fog test was done to double check the simulation and measuring results visually. As the fog test is only a qualitative test, it is not well suited for a detailed comparison with the simulation or measurement results. However, it could be seen that the general path of the airflow was similar to the path shown in the simulation results. The fog test also clearly showed vortices in the airflow.

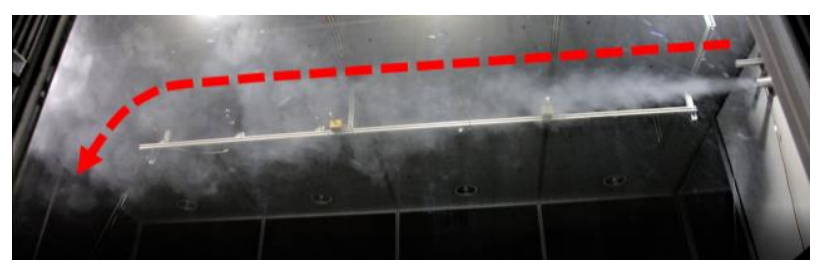

Fig. 11: Airflow visualization with fog

\subsubsection{Comparison with the measurement results}

The measurement results of the three two-dimensional sensors are shown in Figure $12 \mathrm{a}-\mathrm{c}$. The figures show the mean air speed values in the XY-plane (dots) at the respective flow directions, their standard deviation and the relative frequency (bars). The directional value is also shown, where an airflow going downwards has a direction of $270^{\circ}$.

The mean speed values of M1, the sensor closest to the nozzle, are highest of all sensors, having a narrow peak at $180^{\circ}$. M2 shows a broader directional deviation and overall lower mean speed values. The mean speed values of M3 are lowest and peak around $270^{\circ}$, which indicates a downward flow.

The measurements of the local airflow direction are shown in the compasses on the left-hand side of the graphs. It shows the averaged measurement of airflow direction (short arrow) as well as the range of values that were measured (outer ring). The intensity of the color in the ring represents a high relative frequency of the measurement. In addition, the long arrow shows the direction calculated by the simulation.

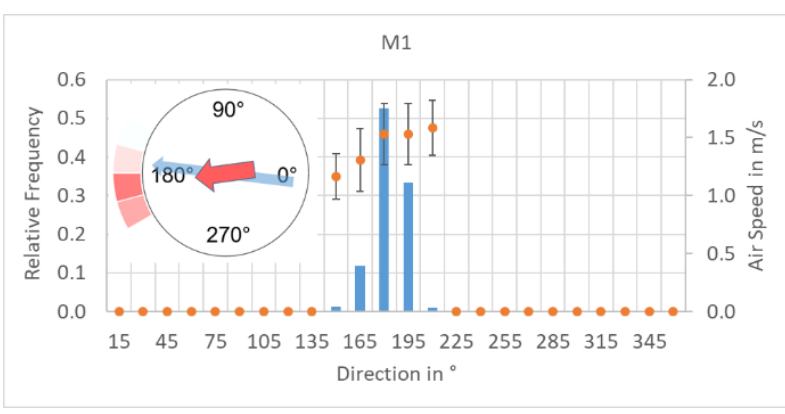

Fig. 12 (a): Measurement results of sensor M1

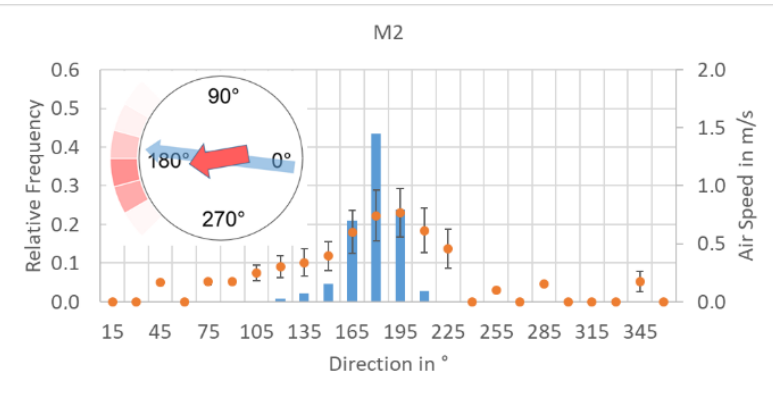

Fig. 12 (b): Measurement results of sensor M2

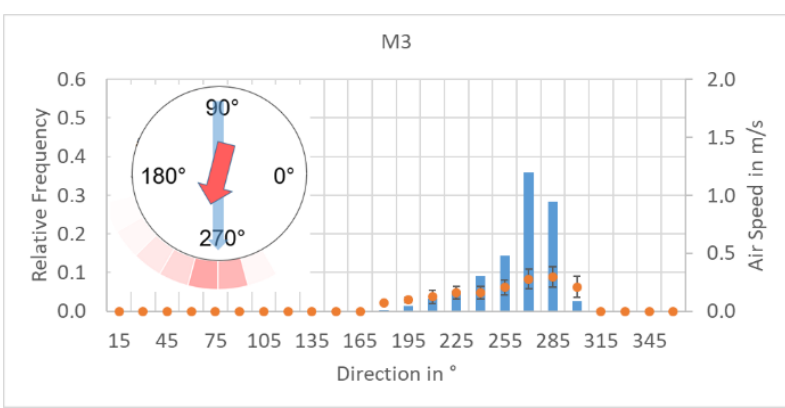

Fig. 12 (c): Measurement results of sensor M3

The following table shows a comparison of the results of the two-dimensional sensors (M), the reference sensors (R) and the Simulation (S). S1, S2 and S3 are samples taken from the simulation data at the position of the twodimensional sensors.

Table 1: Comparison of measurement and simulation

\begin{tabular}{|l|l|c|c|c|}
\hline \multicolumn{2}{|c|}{ Position 1 } & M1 & - & S1 \\
\hline Speed & $m s^{-1}$ & 1.50 & - & 1.48 \\
\hline Direction & $\circ$ & 184 & - & 178 \\
\hline \multicolumn{2}{|c|}{ Position 2 } & M2 & R2 & S2 \\
\hline Speed & $m s^{-1}$ & 0.68 & 0.80 & 0.62 \\
\hline Direction & $\circ$ & 187 & - & 177 \\
\hline \multicolumn{2}{|c|}{ Position 3 } & M3 & R3 & S3 \\
\hline Speed & $m s^{-1}$ & 0.25 & 0.21 & 0.23 \\
\hline Direction & $\circ$ & 251 & - & 269 \\
\hline
\end{tabular}


It is notable that the reference sensors R2 and R3 do not have any entries for the directional measurement. This is because the reference sensors are omnidirectional anemometers without the capability of measuring the direction of the airflow.

The deviations between the mean air speed values of the reference sensors and the two-dimensional sensors lie between 0.04 and $0.12 \mathrm{~m} / \mathrm{s}$. The deviations between the two-dimensional sensor and the simulation vary between 0.02 and $0.06 \mathrm{~m} / \mathrm{s}$. The deviations between the measured and the simulated airflow directions vary between $6^{\circ}$ and $18^{\circ}$.

One reason for this deviation is the measurement uncertainty of both sensors. Another reason for the deviation is the different positioning of the sensors. To avoid the interference during the measurement from other sensors there is a minimum distance of $0.10 \mathrm{~m}$ between the two-dimensional sensor and reference sensor. In addition, the z-component of the velocity can have an influence on the measurement of the two-dimensional sensor, whereas the reference anemometer is an omnidirectional sensor. Compared to the simulation, the two-dimensional sensors show matching results.

The deviations of indicated flow direction between simulation and measurement could be caused by the measurement uncertainty of the two-dimensional sensor $\left( \pm 6^{\circ}\right)$ and by the local fluctuations and turbulence of the airflow. The compasses in Figure 12 show the range of fluctuation of the measurement and their frequency. That means the simulation result is always in between the measuring results of the sensors. A deviation of $6^{\circ}$ to $18^{\circ}$ is acceptable for most real applications of the sensor. The uncertainty of the directional measuring might also be improved in future developments.

\subsection{Heat plume}

The measurements show how a heat plume develops within ten minutes. Figure 13 shows the measured mean air speed values of the reference anemometers (grey bars) and the mean air speed values measured with the twodimensional sensor (black bars). At the start of the experiment, the radiator was switched on with an electric power of $400 \mathrm{~W}$. The values of the reference sensor and the two-dimensional sensor show the same tendency. The maximum deviation between the two sensors is $0.07 \mathrm{~m} / \mathrm{s}$.

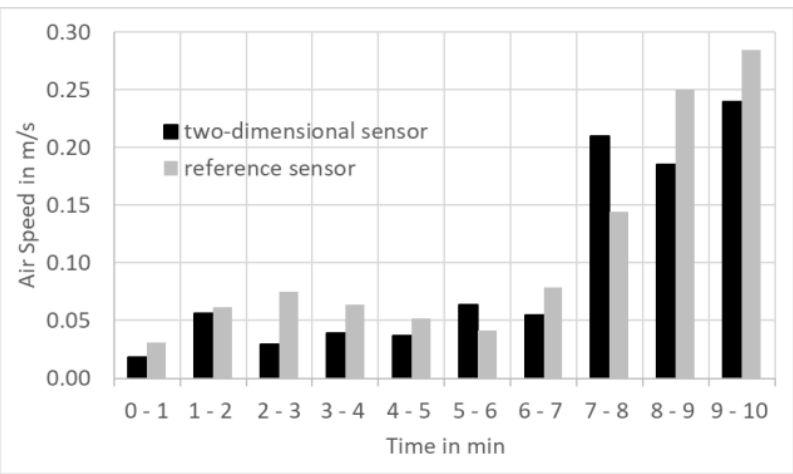

Fig. 13: Measurement results of the heat plume experiment
Figure 14 shows the mean velocity vectors from minute zero to ten, averaged over each minute. The measurement of the flow direction indicates that the air flows upwards, as expected.

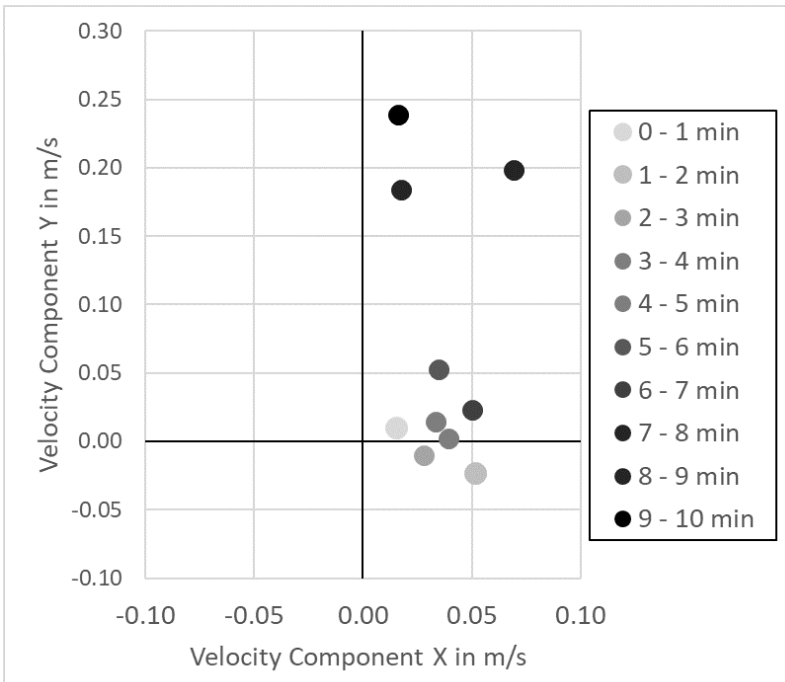

Fig. 14: Plot of the measured velocity vectors of the heat plume experiment.

During the measurements, a fog test was done again to compare the results visually. This test result can be seen in Figure 15. The fog followed the heat plume above the radiator.

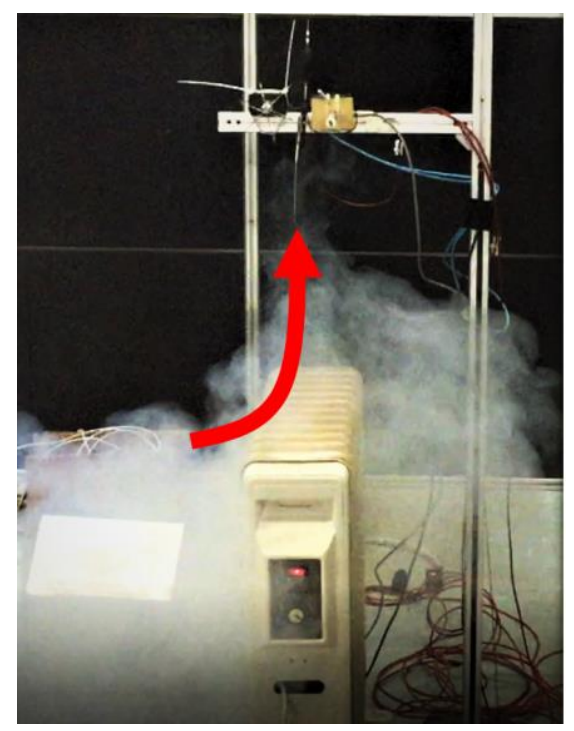

Fig. 15: Heat plumes visualization above a radiator

It should be noted that the measurement location of the two sensors is not exactly the same. Similarities of the measurements of the two sensors stem solely from the symmetrical setup.

\section{Conclusions}

These two application-oriented experiments showed that the main airflow in a ventilated room as well as the buoyant plume from a heat source could be monitored with the newly developed sensors. This means that 
unexpected airflows, e. g. downdraughts can be indicated and corrected in time with suitable control measures (such as the adjustment of the inlet air speed and angle, regulation of the heat output of the radiator, etc.).

Since the sensor is developed for two-dimensional measurement, the z-axis component of the velocity may interfere with measurements. Therefore, the main airflow path must be predicted first and the placement of the sensors must be carefully determined. Even so, it can be seen that the sensor has great application potential in HVAC diagnostics and controls, post occupancy evaluation, natural ventilation design and optimization, cleanroom monitoring, etc. The two-dimensional sensor expands the principle of bi-directional airflow measuring by one dimension. Therefore, it can be used for all applications of bi-directional sensors. By measuring in one additional dimension, more information about the airflow is acquired.

However, there is also much room for development and improvement. Optimization of design and manufacturing of the sensor, development of evaluation software and wireless networking, improvement of calibration test bench and calibration procedure as well as the various application scenarios will be the research focuses in the future.

This research was supported by the Federal Ministry of Economic Affairs and Energy (BMWi) and Industrial Collective Research (IGF). We thank the HLK Stuttgart GmbH who provided insight and expertise that greatly assisted the research. We also thank our research partner, the Hahn-Schickard Society for Applied Research.

\section{References}

1. EPBD 2018, Energy Performance of Buildings Directive (2018)

2. VDI 3803, Air-conditioning - Structural and technical principles - Central air-conditioning systems (2018)

3. ISO 7730, Ergonomics of the thermal environment (2006)

4. P.O. Fanger, A.K. Melikov, H. Hanzawa, J. Ring, Energy and Buildings 12, 21-39 (1988)

5. C. Kaup, HLH 62, 100-104 (2011)

6. M.H. Sherman, Efficacy of Intermittent Ventilation for Providing Acceptable Indoor Air Quality (2006)

7. E. Fiedler, DIN EN ISO 7730 in practice, TGA congress (2016)

8. ISO 7726, Ergonomics of the thermal environment, Instruments for measuring physical quantities (2002)

9. M. Hurnik, M. Blaszczok, Z. Popiolek, Data in Brief 5, 213-217 (2015) (LDA)

10. X. Cao, J. Liu, N. Jiang, Q. Chen, Energy and Buildings 69, 367-380 (2014) (PIV)

11. H. Koskela, J. Heikkinen, R. Niemelä, T. Hautalampi, Building and Environment 36, 247-255 (2001)
12. H. Koskela, H. Häggblom, R. Kosonen, M. Ruponen, HVAC\&R Research 18, 723-736 (2012)

13. Dantec Dynamics A/S, Product documents: Dualsensor miniature wire probes

14. HS-VS, Internal Report

15. Y. Wang, M. Müller, K. Stergiaropoulos, Roomvent \& Ventilation, 355-360 (2018)

16. ISO 16622, Meteorology - Sonic anemometers/ thermometers - acceptance test methods for mean wind measurements (2003) 